\title{
Associations between breakfast consumption, attitudes towards breakfast and physical activity in adolescents
}

\author{
S. Kennedy ${ }^{1}$, L. Ryan ${ }^{2}$ and M.E. Clegg ${ }^{1}$ \\ ${ }^{1}$ Functional Food Centre, Department of Sport and Health Sciences, Oxford Brookes University, Gipsy Lane, Oxford, \\ OX3 OBP and ${ }^{2}$ Department of Nutrition and Dietetics, Faculty of Medicine, Nursing and Health Sciences, Monash \\ University, 264 Ferntree Gully Road, VIC 3168, Australia.
}

Regular breakfast consumption has been associated with children and adolescents having a lower body mass index ${ }^{(1)}$, positive effects on cognitive performance ${ }^{(2)}$, a better overall diet quality and being more physically active ${ }^{(1)}$. However, as children progress to adolescence they become more likely to skip breakfast ${ }^{(3)}$ and physical activity levels decline, particularly in girls ${ }^{(4)}$. Adolescence is a critical time point as habits and attitudes towards healthy behaviours developed at this age are likely to track into adulthood ${ }^{(5)}$. Health behaviours are suggested to cluster in individuals ${ }^{(6)}$, therefore identifying attitudes in adolescents that promote or create barriers to healthful behaviour presents an opportunity to investigate the potential determinants of breakfast behaviours.

The aim of the present study was to examine associations between the frequency of breakfast consumption, attitudes towards breakfast and physical activity levels in adolescents.

Fifty-six schools in Oxfordshire were invited to complete a questionnaire investigating the dietary habits and physical activity patterns of students aged 13 to 17 years. While this is an ongoing study, responses to date were assessed for: self-reported physical activity (PA) levels (PA questionnaire for adolescents; PAQ-A ${ }^{(7)}$ ), agreement of statements measuring attitude towards breakfast based on a previously validated attitude scale ${ }^{(8)}$ and breakfast frequency.

140 participants ( $n=109$ females, $n=23$ male, $n=8$ undisclosed) reported breakfast frequency as everyday $(47 \%), 5-6$ days $(10 \%)$, 3-4 days (13\%), 1-2 days (17\%) and never (13\%). Mean attitude scores (range 1 (negative) to 5 (positive)) and standard deviations (SD) were calculated for each attitude measure and compared by breakfast frequency.

\begin{tabular}{|c|c|c|c|c|c|c|c|c|c|}
\hline \multirow{3}{*}{$\begin{array}{l}\text { Breakfast } \\
\text { frequency }\end{array}$} & \multirow[b]{3}{*}{$\mathrm{n}$} & & & \multicolumn{6}{|c|}{ Attitudes towards breakfast } \\
\hline & & \multicolumn{2}{|c|}{$\begin{array}{l}\text { Usually snack at } \\
\text { morning break }\end{array}$} & \multicolumn{2}{|c|}{$\begin{array}{l}\text { Rather snack than have } \\
\text { breakfast }\end{array}$} & \multicolumn{2}{|c|}{$\begin{array}{l}\text { Usually eat a healthy } \\
\text { breakfast }\end{array}$} & \multicolumn{2}{|c|}{$\begin{array}{l}\text { Feel ok without } \\
\text { breakfast }\end{array}$} \\
\hline & & Mean & SD & Mean & SD & Mean & SD & Mean & SD \\
\hline never & 18 & $2 \cdot 5$ & $1 \cdot 33$ & $2 \cdot 5$ & 1.42 & $2 \cdot 5$ & $1 \cdot 32$ & $2 \cdot 0$ & 1.06 \\
\hline 1-2 days & 23 & $2 \cdot 1$ & $1 \cdot 37$ & $2 \cdot 3$ & $1 \cdot 13$ & $3 \cdot 0$ & $1 \cdot 25$ & $2 \cdot 4$ & $1 \cdot 32$ \\
\hline 3-4 days & 18 & $2 \cdot 7$ & $0 \cdot 97$ & $2 \cdot 8$ & 1.00 & $3 \cdot 7$ & $0 \cdot 83$ & $2 \cdot 2$ & $1 \cdot 11$ \\
\hline 5-6 days & 14 & $3 \cdot 9$ & $1 \cdot 17$ & $3 \cdot 5$ & $1 \cdot 16$ & $3 \cdot 6$ & $1 \cdot 16$ & $2 \cdot 9$ & $1 \cdot 23$ \\
\hline everyday & 67 & $4 \cdot 4$ & $0 \cdot 90$ & $4 \cdot 4$ & 0.99 & $3 \cdot 9$ & 1.03 & $3 \cdot 8$ & $1 \cdot 21$ \\
\hline
\end{tabular}

Pearson's correlation coefficients were calculated between breakfast frequency, attitudes towards breakfast and PAQ-A scores. There were significant positive correlations $(P<0 \cdot 05)$ between PAQ-A scores and breakfast frequency $(r=0 \cdot 2)$ and between attitudes towards breakfast and breakfast frequency $(P<0 \cdot 01)$ with the highest correlations observed between breakfast frequency and attitudes towards snacking behaviours. Breakfast skippers reported greater associations between preferring to snack at morning break and usually snacking at morning break ( $r=0.6$ and $r=0.6$ consecutively).

These preliminary findings suggest associations between breakfast frequencies, attitudes towards breakfast and physical activity levels presenting an opportunity to improve understanding of the determinants of adolescent's breakfast habits.

1. Rampersaud GC, Pereira MA, Girard BL et al. (2005) J Am Diet Assoc 105, 743-60.

2. Hoyland A, Dye L \& Lawton CL (2009) Nutr Res Rev 22, 220-43.

3. Balding A \& Regis D (2013) Young People into 2013 - The Health Related Behaviour Questionnaire.

4. Health \& Social Care Information Centre (2014) Statistics on Obesity, Physical Activity and Diet: England.

5. Conner M, Norman P \& Bell R (2002) Health Psychol 21, 194-201.

6. Pearson N, Atkin A, Biddle S et al. (2009) Int J Behav Nutr Phys Act 6, 45.

7. Kowalski KC, Crocker PR \& Donen RM (2004) The PAQ-A Physical Activity Questionnaire Manual.

8. Tapper K, Murphy S, Lynch R et al. (2008) Eur J Clin Nutr 62, 511-8. 